\title{
THE STUDY ON HEIGHT INFORMATION EXTRACTION OF CULTURAL FEATURES IN REMOTE SENSING IMAGES BASED ON SHADOW AREAS
}

\author{
Zhang Bao-ming Guo Hai-tao Lu Jun Liu Zhi-qing He Hong \\ Institute of Surveying and Mapping Zhengzhou,66 Mid-Longhai Road, Zhengzhou, China, 450052
}

Zbm2003@163.com

KEY WORDS: Cultural Features, Photogrammetry, Regional Segmentation, Shadow Area, Sun Altitude Angle

\begin{abstract}
:
Cultural feature is important element in geospatial information library and the height information is important information of cultural features. The existences of the height information and its precision have direct influence over topographic map, especially the quality of large-scale and medium-scale topographic map, and the level of surveying and mapping support. There are a lot of methods about height information extraction, in which the main methods are ground survey (field direct measurement)、 spatial sensor and photogrammetric ways. However, automatic extraction is very tough. This paper has had an emphasis on segmentation algorithm on shadow areas under multiple constraints and realized automatic extraction of height information by using shadow. Binarization image can be obtained using gray threshold estimated under the multiple constraints. On the interesting area, spot elimination and region splitting are made. After region labeling and non-shadowed regions elimination, shadow area of cultural features can be found. Then height of the cultural features can be calculated using shadow length, sun altitude angle, azimuth angle, and sensor altitude angle, azimuth angle. A great many of experiments have shown that mean square error of the height information of cultural features extraction is close to 2 meter and automatic extraction rate is close to $70 \%$.
\end{abstract}

\section{INTRODUCTION}

With the development of urban construction, more and more high-rise buildings appeared in cities. So in the decisionmakings of city planning, economy affairs and military policies, the height information of tall building has been a necessary parameter. The existences of the height information and its precision have direct influence over topographic map, especially the quality of large-scale and medium-scale topographic map, and the level of surveying and mapping support. There are a lot of methods about height information extraction, in which the main methods are ground survey (field direct measurement)、 spatial sensor and photogram metric ways.

In the field of photogrammetry, basically there are three methods to extract the height information of cultural features: making use of single remote sensing images multichannel stereo-images and multisource data. Researchers in China have deeply investigated the extraction of the height information by using stereo images. However, both domestic and foreign researchers seldom devoted themselves to the research of the extraction of height information based on the shadow, and this is a real potential technique developing into automatization. This paper has had an emphasis on segmentation algorithm on shadow areas under multiple constraints and realized automatic extraction of height information by using shadow. Experimental results proved the feasibility and practicality of this method.

\section{THE BUILDING SHADOW EXTRACTION UNDER MULTI-CONSTRAINTS}

Shadow is inescapable in remote sensing pictures. With the spread of high definition images, how to deal with the shadow of features, became a tough problem and a desiderata in the filed of remote sensing image processing. However, it can provide much related information (such as the shape, relative position, surface character) of the object, which generates the shadow (Barnes et al, 1999; Ortega et al, 1998; Yang et $\mathrm{al}, 2002)$. So, it is important to research in shadow extraction.

\subsection{Two Directions Determination}

Two directions determination means deciding the direction of the building vertical edges and that of the shadow edges of vertical buildings by the auxiliary information.

In projective geometry, parallel lines in real scene converge to one point in image, which is called vanishing-point. Since all vertical edges of the building are parallel, they must have the intersection point in the image. In the frame image, the direction of the building vertical edge can be decided through the image nadir, whose coordinates can be calculated through the image's exterior elements. For the other types of images, the determination of the building vertical edge is relatively more complex. Take the IKONOS image for example, while approximately vertical photography is done, the direction of the vertical edge can be approximately decided by the center point of each scan line. Other conditions, the direction of the vertical edge can be approximately decided by the angle elements of exterior orientation.

Major buildings' shadows, especially the vertical building's shadows have the same direction in the image, which is also just the direction of sunlight. In other words, the direction of the shadow edges of vertical buildings accord with the sunlight direction. So the edge of shadow at the sunlight direction can be extracted first, and then building shadow can be extracted by 
calculating grey pixels along the structure line statically. Most satellite remote sensing data provide sun azimuth and zenith angle in the header file. For the aero remote sensing image, the direction of sunlight can be decided by the imaging time and the position of the imaged area.

\subsection{Edge Detection by Canny Operation}

After the direction of the building vertical edges and that of the shadow edges of vertical buildings are decided, useful edge detectors can be utilized to detect edges along these two directions.

The canny operator is set up upon the optimal detection rule, the optimal detection rule and the mono-response rule. It can achieve good balance between noise restrain and edge detection and is the optimal choice for step edge under white noise contamination (J F Canny,1986).

To avoid interruption from unnecessary edges and facilitate the subsequent edge extraction, the Canny operator is applied at not the all direction but only the direction of the building vertical edges and that of the shadow edges of vertical buildings.

\subsection{Straight Edge Track under Constraints}

Since the Canny operator has detected edges along these two critical directions, the subsequent work is to track and extract these detected edges using the straight edge track algorithm.

For example, if the edge has " $\theta$ " angle to the positive direction of the " $x$ " axis, the slanting ratio of the edge line can be deduced from " $\theta$ ". Suppose the line equation is " $y=k_{0} x+b$ ", then " $b=y-k_{0} x$ ". For any given image, the range of "b" is easily drawn, " $b_{\min } \leq b \leq b_{\max }$ ", but "b" is treated as an integer during real track. The detailed track procedures are listed as follows.

(1) For a given " $b$ " and slanting ratio " $k_{0}$ ", a " $y$ " value can be calculated from the equation " $y=k_{0} x+b$ ". During the first iteration, " $b$ " is set to be " $b_{\min }$ ". Make a judgement on the calculated value " $(\mathrm{x}, \mathrm{y})$ " to see whether the point " $(\mathrm{x}, \mathrm{y})$ " is the feature point. If it is the feature point, note the value as the first value and the current tracking point, else abandon it and increase the number of " $x$ " by one, until find the first feature point.

(2) Increase the " $x$ " by one, you can get another point "(x,y)". Under if the circumstance that the newly tracking point is known, the distance "D1" between point "(x,y)" and the newly tracking point is larger than the threshold value " $\mathrm{d} 1$ " and the distance "D2" between the first tracking point and the newly tracking point is larger than the threshold value " $\mathrm{d} 2$ ", the line between the first tracking point and the current tracking point is traced out and is saved. If "D2" is no larger than threshold "d2", abandon the line. As long as "D1" is larger than the threshold value " $\mathrm{d} 1$ ", set the marking "flag=1" and be prepared to trace another line. Make a judgement on the point $(\mathrm{x}, \mathrm{y})$ and its eight neighbourhood points to see if they are feature points. As long as one of these nine points is the feature point, take it as the current tracking point. Moreover, if " $\mathrm{flag}=1$ " is satisfied at the same time, set this new feature point as the starting point of the new line and make "flag=0".

(3) Continue step (2) until the value of " $x$ " equals to the width of the image. Trace out all the lines (" $k=k_{0}, \quad b=b$ ") whose length are greater than the threshold value "d2".

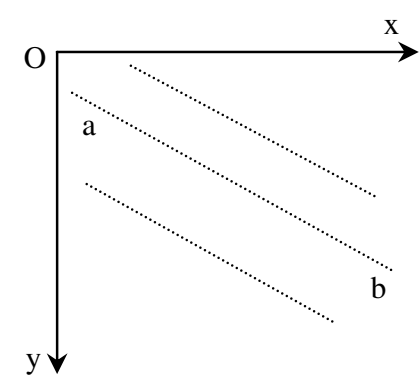

Figure 1. Straight edge track under constraints

(4) Increment b by one, and repeat step (1), (2) and (3) to trace out all the lines with " $k=k_{0}$ " whose length are greater than the threshold "d2".

(5) Repeat step (4) until " $b=b_{\max }$ ". Trace out all the lines with " $k=k_{0}$ " whose length is greater than the threshold " $\mathrm{d} 2$ ". All the edges can be successfully traced out, whose length are great the threshold value along the direction of the building vertical edges and that of the shadow edges of vertical buildings.

\subsection{Assessment on the Shadow Division Threshold}

After tracking out the shadow edge of the vertical buildings using the operator of "the straight edge track under constraints", the shadow division threshold is assessed. This procedure is composed of two steps which are eliminated the pseudo shadow edges and statistically calculating the grey pixels along the shadow edge.

(1) Elimination of the pseudo shadow edge.

The line edges at the vertical building extracted out in the above procedure are not all the shadow edges in fact which maybe roads at this direction, or the building edge. To assess the shadow separation threshold accurately, pseudo shadow edges must be eliminated out. Four constraints are combined to eliminate pseudo shadow edges and keep real ones.

The first constraint is that great difference should exist between grey values of pixels at two sides along the shadow edges. In the experiment the threshold for the pixel grey value difference is set to be one half the image grey variances. The edges with similar grey pixel at two sides will be eliminated out using the constraint, for example, the linear road.

The second constraint deems that the length of the shadow edge should not be too large whose threshold value is set to be the ratio between the real distance in the field and size of image pixel. Through this constraint too long linear roads and railroads will be excluded out.

The third constraint supposes that the shadow edges, especially that of tall buildings will not be too over-dense. In the experiment it is deemed that there will not exist more than two edges in the certain distance at the direction right to the shadow direction. Over dense edges, such as roads and railroads in the high resolution image can be excluded out through this approach.

The fourth constraint is the context information, which assumes the building vertical edges exist near the anti-sun side of the shadow edges, especially for the tall buildings. This constraint can be used as auxiliary rule to exclude pseudo shadow edge which is most advantageous for tall buildings. Moreover the grey constraint that the grey pixel value near the shadow edge at the anti-sun side is generally larger than that of the shadow can also be used to exclude pseudo shadow edge. 
These four constraints mainly consider the grey constraint, the geometrical constraint and context constraint.

(2) Statistical calculation of pixel grey values at one side of the shadow edge.

After exclusion of pseudo shadow edges, the remained edges are mostly that of shadow, which are called the supposed edges. Statistical calculation is reformed on the pixels with smaller grey values in the certain width along the supposed edge. The width is related to the size of the pixel. Try to find out the peak grey level $g_{\max }$ in the histogram, and calculate the total grey variance $\sigma_{0}$. Then treat the pixels whose grey values are smaller than $g_{\text {max }}+k_{1} \sigma_{0}\left(k_{1}=1.5\right.$ in the experiment $)$ as samples and do further calculation of the average value $\mu$ and the variance $\sigma$ on these samples. In this approach, the false shadow pixels along the edge will be excluded out and only the true shadow pixels are remained for statistical calculation. Finally the grey threshold for the shadow separation is decided as $\mu+k \sigma\left(k_{1}=1.6\right.$ in the experiment).

\subsection{Verification of Shadow Extraction Results}

Binary separation is performed area based on the above assessed grey threshold. But perhaps the separated interested area is composed of not only the shadows but also the water areas and vegetations with smaller grey. Therefore false shadow areas need to be further excluded out through the verification of the extraction results. After validation, only real shadow areas will be kept. Before validation, speckle reduction and region division will be carried out firstly in the AOI (Area of interesting). Theoretically, region division can be carried out from any point in the adjacent area to the shadow and towards any direction. But it is difficult to realize in algorithm. In the experiment, region division mainly starts from the direction of sunlight (in Figure 2). Then region marking is done to find the belonging of the pixels and give the peculiar sign of the region. The 8-neighbourhood region marking algorithm is taken. Finally the contour of the region is extracted out.

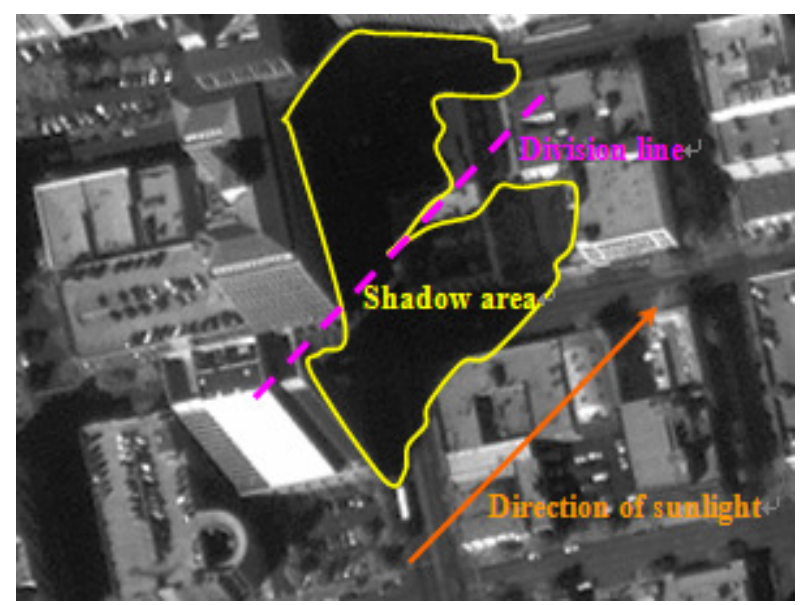

Figure 2. Division for the adjacent areas of shadow

There are three rules for judging whether the region belongs to the shadow area. The first rule is that the area of the region is not too large and the length of the region along the sunlit direction is too long. The threshold of the area is decided upon the image resolution. The threshold of the length takes the pixels length corresponding to room in the field. The second rule is that certain boundaries of the region accord with the sunlit direction and there exists extracted building vertical edges in the anti-sunlit side near the region. The 3rd rule is that the average grey value of certain pixels in the anti-sunlit side near the region is obviously larger than the average grey value of the shadow.

\section{OBTAINING THE HEIGHT INFORMATION OF CULTURE FEATURES VIA SHADOW}

Making use of the shadow in images or utilizing the information provided by feature projection is a traditional method to accomplish the estimation of the elevation information of culture features.

3.1 The extraction principle of the height information of culture features in tower type

If $\Delta h$ is the height of the cultural feature's vertex, and $\mathrm{L}$ is the length of the projection of the vertex. By utilizing the distance between the ground base and corresponding points on the shadow, the value of $\mathrm{L}$ can be calculated and $\theta$ is the sun altitude angle. The height of the cultural features in tower type can be calculated:

$$
\Delta h=L \times \operatorname{tg} \theta
$$

\subsection{The extraction principle of the height information of} other isolated culture features

(1) The corresponding point is found by making use of the culture features and its shadow extraction. The shadow length is measured. The height information of culture features is obtained by formula (1).

(2)When the sun and the sensor are at the same side of the culture feature

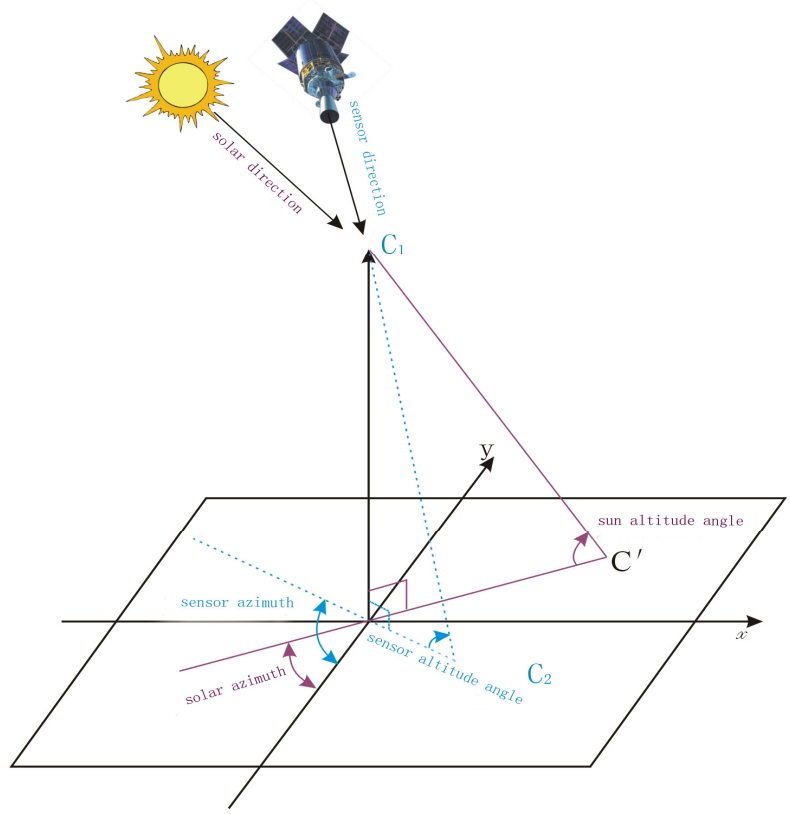

Figur3 The geometrical relationships of angle point projection and shadows 
In this condition, sun shadow and observational shadow are partly shielded by each other. If $\theta$ is solar altitude and $\beta$ is sun altitude angle. When at the condition of $\theta<\beta$, the angle points of culture features in the image is actually the point projected to ground from the direction of sensor. The boundary close to shadow is not the real ground base of the culture feature in remote sensing image. So, on the boundary, for the part of angle point, it is tough to find the base point. Then, by directly using the link lines between the angle points and corresponding point on the shadow, the geometrical relationship is restored between the points in the images and sun altitude angle、 solar azimuth 、 sensor altitude and sensor azimuth, and then the height information can be calculated. The link line is satisfied with the geometrical relationships showed in Figur3.

As shown from Figur3, angle point $C_{1}$ is showed as $C_{2}$ in the image and $\mathrm{C}_{2}$ is the position of angle point in the map. The idiographic position of $\mathrm{C}_{2}$ is related to sensor altitude $\theta$ and sensor azimuth angle $\psi$.In Figur3, the straight line $\mathrm{C}_{1} \mathrm{C}$ is upright against to ground. The distance $\mathrm{L}$ between $\mathrm{C}_{2}$ and $\mathrm{C}^{\prime}$ is measured. From above-mentioned geometrical relationships, the distance $l_{1}=\Delta h \times \operatorname{ctg}|\beta|$ between $\mathrm{C}$ and $\mathrm{C}_{2}$, between $\mathrm{C}$ and $\mathrm{C}^{\prime}$, $l_{2}=\Delta h \times \operatorname{ctg}|\theta|$. The angle between solar azimuth and sensor azimuth $\lambda=\angle C_{2} C C^{\prime}=\Phi-\Psi$. So, in the triangle $\mathrm{C}_{2} \mathrm{CC}^{\prime}$, according to law of cosines:

$$
L^{2}=l_{1}^{2}+l_{2}^{2}-2 l_{1} l_{1} \cos \angle C_{2} \mathrm{C}^{\prime}
$$

Namely,

$$
L^{2}=\Delta h^{2} \times\left(\operatorname{ctg}^{2}|\beta|+\operatorname{ctg}^{2}|\theta|-2 \operatorname{ctg}|\beta| \times \operatorname{ctg}|\theta| \times \cos \lambda\right)
$$

So, the height of angle point $\mathrm{C}$ can be calculated as:

$$
\Delta h=\frac{L}{\sqrt{\operatorname{ctg}^{2}|\beta|+\operatorname{ctg}^{2}|\theta|-2 \operatorname{ctg}|\beta| \times c t g|\theta| \times \cos \lambda}}
$$

By using the above geometrical relationships, the distance $\mathrm{L}$ is measured between the projection point of angle point and corresponding point on the shadow, then the actual height can be calculated.

When $\theta>\beta$, since the gradient of the sensor is bigger than that of sun, the point falling to the ground of feature boundary will shield most of the shadow. On this condition, we can only depend on the shadow of critical angle point to calculate the height and the above-mentioned several methods are unsuitable. Other methods should be found. But as sensor altitude angle is usually big, if solar altitude angle is bigger than it, the shadow area is so small that it will be meaningless for a further research.

\section{EXPERIMENT AND CONCLUSION}

In Experiment, the building shadow extraction is done with several IKONOS images and high resolution (about $1 \mathrm{~m}$ ) aerial images. The shadow extraction results of one IKONOS image are shown in Figure 4. Picture (a) in Figure 4 is an original IKONOS image. Picture (b) is the shadow extraction results. Results of the experiment show that this approach is effective and robust to extract building shadow, especially for the building shadow in high resolution images.

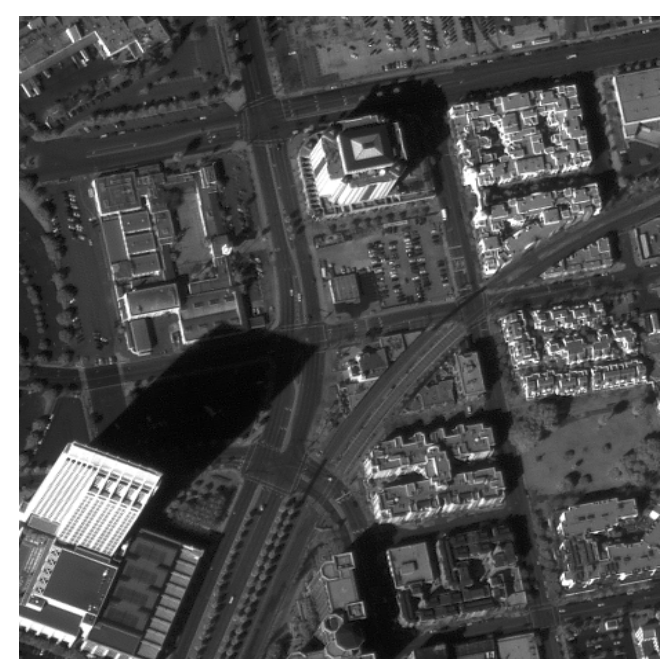

(a) Original IKONOS image

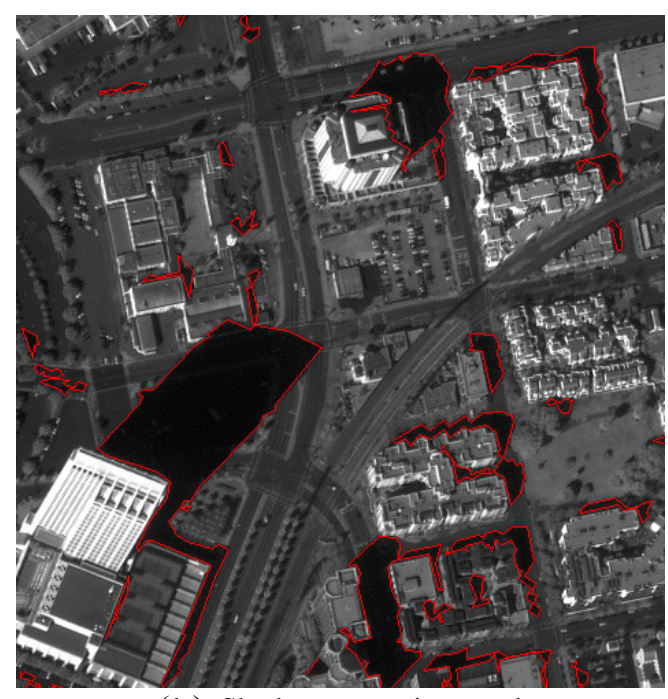

(b) Shadow extraction result

Figure4 The shadow extraction results of one IKONOS image

On the base of shadow extraction, height information extraction is done with the IKONOS image, and the result is shown in List1. The measurement mean square error is $2.08 \mathrm{~m}$.

From List1, it can be concluded that the precision of height information extraction (based on shadow extraction) is better than $3 \mathrm{~m}$. There are approximately 148 culture features(higher than $5 \mathrm{~m}$ ). 102 features' height information can be automatically worked out, the rate of automatic extraction is $68.9 \%$, better than $60 \%$. The main reason of failure extractions is that some shadow cast to some other culture features or some features in uneven areas, making it impossible to measure the actual longness precisely.

\section{REFERENCES:}

Barnes N. and Liu Z.Q,1999. Knowledge-based shape from shading. Int. J. of Pattern Recognition and Artificial Intelligence, 13(1), pp.1-23. 
J F Canny,1986. A computational approach to edge detection. IEEE Transactions on Pattern Analysis and Machine Intelligence, $8(6)$.

Ortega A.and Shah M, 1998. From shape from shading to object recognition. Int. J. of Pattern Recognition and Artificial Intelligence, 12(2), pp.191-208.
Yang Yijun, Zhao Rongchun and Jiang Wenbing, 2002. Detection of shadow areas from aerial imagery. Signal Processing,18(3),pp.228-232.

List 1 The culture feature height information extraction(based on shadow extraction ) results of one IKONOS image

\begin{tabular}{|c|c|c|c|c|c|}
\hline $\begin{array}{c}\text { Feature } \\
\text { Number }\end{array}$ & $\begin{array}{c}\text { Feature } \\
\text { Height }(\mathrm{m})\end{array}$ & $\begin{array}{c}\text { Feature } \\
\text { Height By Man- } \\
\text { machine Interaction 1 } \\
(\mathrm{m})\end{array}$ & $\begin{array}{c}\text { Error 1 } \\
(\mathrm{m})\end{array}$ & $\begin{array}{c}\text { Feature } \\
\text { Height By Man- } \\
\text { machine Interaction 2 } \\
(\mathrm{m})\end{array}$ & $\begin{array}{c}\text { Error 2 } \\
(\mathrm{m})\end{array}$ \\
\hline 1 & 144.5 & 142.1 & 2.4 & 143.7 & 0.8 \\
\hline 2 & 60.4 & 61.5 & 1.1 & 58.2 & 2.2 \\
\hline 3 & 43.5 & 44.8 & 1.3 & 43.5 & 0.0 \\
\hline 4 & 15.9 & 14.6 & 1.3 & 14.1 & 1.8 \\
\hline 5 & 125.5 & 121.9 & 3.6 & 121.5 & 4.0 \\
\hline 6 & 22.8 & 21.1 & 1.7 & 21.7 & 1.1 \\
\hline 7 & 24.5 & 23.8 & 0.7 & 22.4 & 2.1 \\
\hline
\end{tabular}

